\title{
Grasping the Nettle of L2 Idiomaticity Puzzle: The Case of Idiom Identification and Comprehension During L2 Reading by Greek Learners of English
}

\author{
Eirene C. Katsarou \\ State EFL Teacher \\ ekatsai@otenet.gr
}

\section{Abstract}

This study investigates the extent of identification and comprehension success achieved by Greek EFL high school students when encountering phrasal idioms during reading English as a foreign language. The study also examines the relationship of the above with five idiom type features, i.e. (i) contextual guessability, (ii) frequency of words in the idiom, (iii) interlingual similarity, (iv) transparency and ( $v$ ) existence of an idiom in Greek and four learner factors: (i) L2 language proficiency, (ii) gender, (iii) motivation and (iv) field-independence/dependence. The results indicate that overall scores for $\mathrm{L} 2$ idiom identification and comprehension were remarkably low. Successful L2 idiom identification was found to correlate strongly but negatively both with frequency of occurrence of the component words of the idioms and with interlingual similarity while L2 idiom comprehension was found to correlate significantly positively with interlingual similarity only. No significant correlations were obtained between any of the four learner-related variables and the two main variables of $L 2$ idiom identification and comprehension. Some pedagogical implications are also offered that mainly consist the practical implications of the study in terms of instructional practices and methodology employed in the teaching of English idioms in predominantly EFL contexts.

\section{Introduction: Two Problems for Idiom Research}

Common lexical problems in the process of $L 2$ reading comprehension caused by limited vocabulary knowledge often tend to be associated with the word as the primary unit. However, previous research findings lead us to expect that idioms can also be a source of potential communication breakdown in L2

(cc) BY-NC-ND 
reading comprehension due to two main reasons (a) their misidentification and, (b) their subsequent misinterpretation in a text context.

The issue of misidentification has been researched to some extent, but hardly any of it was done on idioms. Laufer (1989), for instance, investigated deceptive transparency as a factor of difficulty in L2 vocabulary learning and reading comprehension based on students' interpretations of various DT (deceptively transparent) words in a text i.e. words that they look as if they are familiar but are easily misidentified on the part of the learner and thus can lead to the miscomprehension of the text and her results revealed that learners more frequently made errors with DT rather than with non-DT words ( $t=1.67$ $p<0.05$ ) showing little awareness of their ignorance of DT words, a fact which subsequently, significantly affected their reading comprehension $(p=.0001$ level). According to Laufer and Sim (1985:5), misinterpretation mainly arises when the textual knowledge is itself a misinterpretation - i.e. when a word is mistakenly identified - leading to even further

misinterpretation of the information in the text which is finally distorted into a false construct to suit the reader's conviction.

Since idioms are also classified as deceptively transparent words by Laufer (1997), it is suggested that this situation might apply even more to the case of idioms than to single words, as idioms often are made up entirely of words that are separately known to the learner (e.g. pull my leg) who, in his effort to make sense of the text he is reading, may resort to one-for- one translation of the words of an idiomatic expression due to his failure to recognize that he is dealing with an unknown idiom that has to be interpreted as a multi-word lexical expression with a unitary meaning.

\section{A Working Definition of Idiom in English}

Acknowledging the multifarious character of idiomaticity in language, we believe that it would be best to opt for a multi-dimensional definition with each property clearly delineated with the intention to reach a narrow as well as a strictly linguistic definition of 'idiom' as a distinct lexical unit. Given below is a summary of the defining features adopted for the characterization of a pure verbal phrasal idiom as it is currently used for the purposes of the present study:

A pure verbal phrasal idiom is:

1. lexically complex - i.e. it should consist of at least more than one lexical item that can freely occur as single words outside the idiom.

2. grammatically well-formed, and for our study we have chosen to restrict ourselves to the syntactic V+NP type (e.g. kick the bucket).

3. semantically not the result of the compositional function of its constituent parts, although not necessarily totally opaque as some idioms may contain 
a metaphorical element that contributes to their partially transparent meaning.

4. syntactically stable allowing the minimum of transformational potential; in its structure.

5. a 'double exposure' i.e. a pure idiom has both a literal and a non-literal 'face', due to the existence of a literal homonymous counterpart which complements that fact that the syntax of an idiom is non-correlative.

In this study, we opt for a multi-dimensional definition of what we call pure verbal phrasal idiom (Fernando et al 1981:22). Thus:

\section{A pure idiom is a non-literal, grammatically well-formed set expression that consists of more than one familiar lexical items and allows the minimum of syntactic transformational potential in its structure and whose meaning is not a compositional function of its syntactic constituents, but which always has a homonymous literal counterpart.}

In essence, our definition of idiom stresses two key features in particular: a noncorrelative syntax, i.e. the grammatical structure of an idiom does not contribute to the meaning of idiom through a one-to-one literal translation of its word components, thus resulting in non-literalness and homonymity. While Weinreich (1969) and Makkai (1972) choose the property of 'disinformation' or 'potential ambiguity' underlying our criterion (5) as the key feature of an idiom, we regard (3) and (5) as complementary and of equal importance in our definition of pure idiom in view of the delimitation of the term per se as well as of its use in the present study. On the one hand, the consequence of the two features of semantic non-compositionality and literal homonymity in a pure idiom is that the mismatch between the apparent (literal) and the intended signification (often metaphorical) is much greater than is the case with other figures of speech in general.

Our definition has several consequences for what we may find with respect to (i) identification and (ii) comprehension of unknown target idioms. Being more semantically covert than most single words and other fixed expressions (e.g. clichés, collocations, catchphrases, etc), a pure idiom can also be more difficult to identify by a non-native speaker of English. If we accept that the essence of idiomaticity rests on an asymmetry between syntax and sense, then our argument is that the presence of a homonymous literal counterpart complements such asymmetry both structurally and contextually and assigns to idioms the status of being readily 'mistakenly identified' or deceptively transparent lexical items that constitute a major stumbling block in the course of reading comprehension in L2 contexts. Furthermore, where unknown idioms are finally identified, our definition has potential consequences for their subsequent codebreaking and interpretation in text context. In this respect, we claim that the existence of a 
literal counterpart, the regularity of the syntax and the multiword nature of the idioms provide far more internal cues than are available in the case of single unknown words for $L 2$ learners to use in their guessing attempts along with the cues supplied by the surrounding text context.

\section{Research Questions and Hypotheses}

The exploratory nature of the study together with the literature previously reviewed gives rise to questions that have not been fully answered concerning the processes of identification and comprehension of idioms carried out by L2 learners when reading an extended text. Some of the research questions and hypotheses that this study sought to answer were the following:

Research Question 1: What type of variables, idiom-type or learner-related, affect Greek EFL learners' ability to identify unknown target idioms while reading a text in English?

Hypothesis 1: There will be a negative relationship between interlingual similarity and the successful identification of idioms.

In his studies, Liontas (2001) and Liontas (2002) found that different idioms (Post-Lexical Level idioms) were detected more often than the identical or similar idioms. Hence, we can predict that interlingual similarity is a factor that does not contribute to the successful identification of idioms.

Research Question 2: What type of variables, idiom-type or learner-related, affect Greek EFL learners' ability to understand the meaning of unknown target idioms while reading a text in English?

Hypothesis 2: There will be a positive relationship between interlingual similarity and the successful inferencing of idioms.

This hypothesis is based on results by Irujo (1986) and Liontas (2002) who found that L1 interference plays an important role in the identification and comprehension of L2 idioms, especially when the source and the target languages are close to one another in equivalent linguistics terms.

\section{The Main Study}

\subsection{Design}

The nature of the research is to a great extent exploratory with the exception of a few hypotheses. The sample for the main study consisted of $60 \mathrm{EFL}$ students in 
three senior high schools who were either in the first or in the second grade of their studies including an equal number of both males and females of all three levels of English language proficiency, i.e. upper-intermediate, advanced and proficient.

The main variables used in the study include both explanatory (independent) and dependent variables and the study contains a correlational and a repeatedmeasures design that relates idiom types and subject variables with their successful identification and comprehension through inferencing in text context. More specifically, independent variables include (a) learner-related, i.e. (i) L2 language proficiency as measured via Nation's Vocabulary Test (Academic Vocabulary) and Oxford Placement Test (Grammar Part only) as well as on their responses to the background questionnaire with respect to whether they were holders of either the First Certificate in English (FCE) or the Certificate of Advanced English (CAE) or the Certificate of Proficiency in English (CPE). (ii) gender, (iii) motivation as measured in the background questionnaire and (iv) field-independence/-dependence as was tested via the administration of the Greek version of the Group Embedded Figures Test (GEFT) and (b) idiomrelated variables, i.e. (i) type of idiom. The English idioms used in the study were classified into three groups based on (a) degree of semantic transparency, i.e. opaque, semi-transparent and transparent, (b) degree of formal similarity to L1 Greek idioms, i.e. identical L1-L2 idioms, similar L1-L2 idioms and L1 idioms different in form from L2 idioms and (c) existence of a Greek idiom with the same meaning for all idioms. (ii) word frequency and (iii) contextual guessability.

Dependent variables include (i) successful L2 idiom identification in terms of how accurately unknown L2 idioms are identified by Greek EFL learners in the text and (ii) successful L2 idiom comprehension in terms of how accurately Greek EFL learners can infer the meaning of unknown L2 idioms in the English text.

\subsection{Instruments}

Five instruments were used in this study:

(1) The Idiom Identification and Comprehension Task (IICT). In the first part learners were asked to find out the 18 English idioms that were incorporated in the text in a natural way without being signposted as such either by presenting them in bold type letters or underlining them (see Appendix A below). To achieve authenticity in reading for a real purpose, we also provided seven reading comprehension questions to be completed based on the information of the text. An immediate retrospective mini-questionnaire formed the second part of the instrument which was used to elicit information on the inferencing strategies used by L2 learners when trying to guess at the meaning of the unknown idioms in the text as well as to measure their confidence in relation to the accuracy of their guesses on a five-point scale. 
(2) The Academic Level of Nation's (VLT) Vocabulary Levels Tests and (3) the Grammar section of the Oxford Placement Test were used to measure L2 learners' level of language proficiency in English. (4) The Groups Embedded Figures Test (GEFT) was administered to determine learners' cognitive style of field-independence/dependence (FI/FD). (5) A background student questionnaire was used to elicit general information on EFL learners' demographic characteristics, educational background, English language learning experience as well as on their attitudes, interest and motivation towards idioms in EFL.

The 18 idioms included in the study were selected according to the 5 following principles: (i) They had to be unknown to the learners: In a separate section in the mini-questionnaire L2 learners were asked whether or not they already knew an idiom before guessing at its meaning. (ii) Interlingual similarity: 9 idioms were grouped into 3 classes with 3 idioms each based on their formal and semantic degree of similarity to L1 Greek. Thus, three idioms were identical in form and meaning in L1 (Greek) and L2 (English) e.g. to leave a bad taste in one's mouth

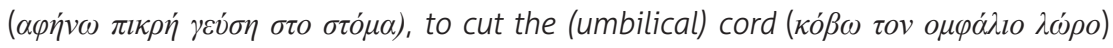

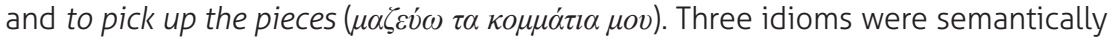
similar and formally similar in L1 (Greek) and L2 (English) e.g. to add fuel to the fire

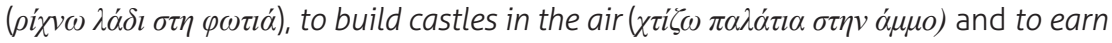

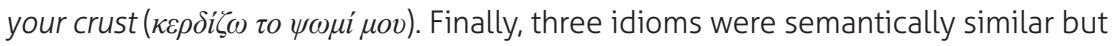
totally different in form in L1 (Greek) and L2 (English) e.g. to ride high in the saddle

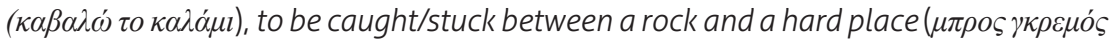

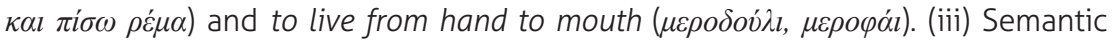
transparency: 9 idioms were grouped into 3 classes with 3 idioms each based on English NS's intuitions on a judgement task as semantically (i) opaque if their component words did not contribute at all to the meaning of the idiom (e.g. to fly off the handle, to play fast and loose, to (not) turn a hair), (ii) semi-transparent when the component words contributed to some extent to their meanings (e.g. to smell a rat, to throw caution to the wind, to clean up your act) and (iii) transparent based on the extent to which their component words contributed to the idiom meaning e.g. via an obvious metaphor (e.g. to rock the boat, to set the wheels in motion, to take the rough with the smooth). These idioms had no idiom equivalence at all in L1 Greek but only corresponded to literal non-fixed expressions (iv) Frequency of use of the component words included in each idiom: The mean frequency score for every idiom was calculated by adding up the number of times each of the content words of an idiom occurred in the BNC corpus and dividing the total by the number of content words in an idiom. (v) Contextual guessability of idioms was determined by giving the modified text to 3 NSs where the 18 target idioms were replaced by blanks and asking them to fill in the blanks in the best possible way by providing either the most plausible verbal idiom or verbal phrase they could think of that fitted the context of the text. 
All instruments were translated in Greek and distributed to the students by the researcher herself in class time in the following order: the Idiom Identification task along with the reading comprehension test, the immediate retrospective mini-questionnaire on idiom guessing strategies and confidence, the Oxford Placement Test, the Vocabulary Levels Test, the GEFT test and the Background questionnaire. The SPSS program version 16.0 was used for the quantitative analysis of the data for the idiom identification and comprehension results.

\subsection{Results for Idiom Identification Success}

\subsubsection{Overall Idiom Identification Success}

With respect to overall success rate in the idiom identification task, figure 1 . displays that Greek EFL learners in this study scored extremely low with a mean response that reaches only $14.75 \%$ of identified unknown idioms only. Idioms that were already known to the subjects of the study were totally discounted from this percent of identification success rate. The low score of idiom identification success is further supported by the distribution of the identification scores that appear to be widely spread $(S D=22.465)$ and positively skewed against the zero percent end of the scale hence not matching the superimposed normal curve. This merely indicates that Greek EFL learners overall tended to score low in the idiom identification task, displaying a prominent failure to identify idioms while reading a text in English as a foreign language.

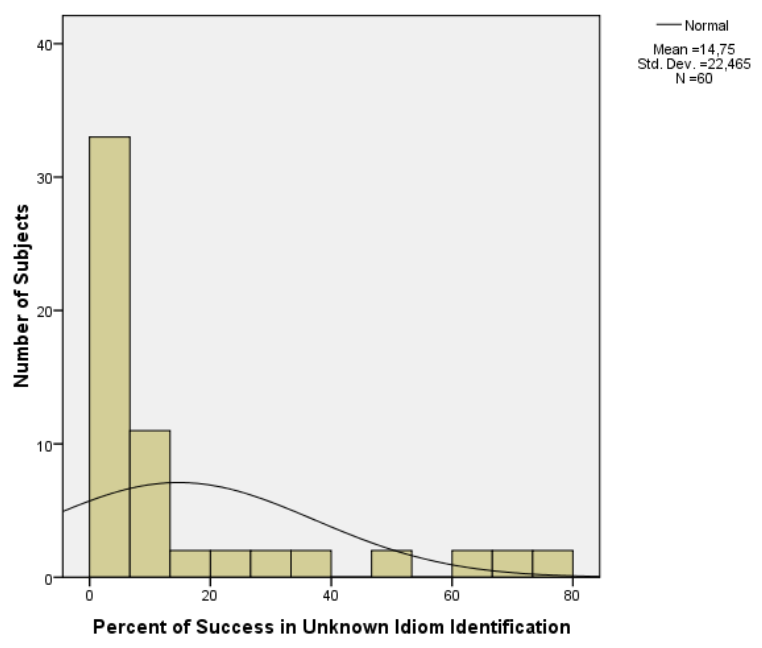

Figure 1. Overall Success in the Idiom Identification Task 
This idiom identification score is even lower when calculated with 'idioms as cases', i.e. $13.12 \%$ and comes in sharp contrast with results of previous studies where the total rate of idiom identification success was: 70.01 in Liontas (2001), 89.99\% in Liontas (2002) and $43.39 \%$ in Dominguez (2008). However, the results of idiom identification success presented in this study can only partly be compared with Liontas (2002) and Dominguez (2008) as both researchers were interested in investigating the process of idiom identification by foreign language learners employing an explicit identification task. The recognition of idioms in Irujo (1986) was rather testing the meaning of the idiom by learners in an ESL context. Our results only partly support Liontas $(2001 ; 2002)$ and Dominguez (2008).

Hypothesis 1 was confirmed, as dissimilar or different idioms in L1 and L2 were detected more often than idioms with a Greek equivalent but nevertheless at a considerably less successful rate, $11.66 \%$, as compared to Liontas (2002) 96.43\% and Dominguez (2008) 53.08\% identification success for different idioms. Obviously, where subjects are directed to consciously identify idioms as the focus of a task as was the case in all these studies above, one might expect more to be spotted than when the idiom identification task is left incidental to the main task set such as for instance a reading comprehension task in our study. Nevertheless, we also believe that the size of the difference in the percent of idiom identification success rate between the two studies and ours is further exaggerated by the fact that our Greek EFL learners seem to have underreported what they identified, as there is a possibility that in cases where they had identified a new idiom but felt that they managed to figure out its meaning with the help of the text context, they did not underline it as such.

S6: ...I recognized some of the idioms but I did not underline them because I did not have any specific problem with them and it was easy for me to understand their meaning based on the meaning of the paragraph and/or context of the sentences before or after the point where the idiom was.

S8: I understood that there were idioms in the text and I underlined some of them. For those I did not underline it was because I could figure out the meaning of the text and the idioms were not really an obstacle. The meaning of the text was also really easy and also the sentence meaning somehow helped to understand the idiom meanings and that's why I did not underline them as unknown. I frequently skipped throughout my reading as I thought that their meaning was not so crucial to the text.

S9: I sort of understood what the text was all about and I didn't pay attention to phrases that did not interfere with my reading... only stopped to one or two points in the text and really wondered what the text meant there, but, on the whole, I tried to use the text context to make sense of what was going on in the passage. 
As can be seen from learners' retrospective comments during interviews and despite clear instructions to underline anything not known prior to reading the text, subjects 6 and 8 did not feel the need to underline idioms they had identified as unknown lexical units as the text context they were resorting to in the form of the paragraph as well as of the immediate sentence where the idiom was could easily give away its meaning. By contrast, where text context offered no available clues to help subjects understand the meaning of idioms and overcome their reading comprehension difficulties, learners tended to skip the phrases which they thought were not central to the meaning of the text such as in the case of subjects 8 and 9 above.

Such a difference in results can also in part be attributed to the more artificial experimental conditions under which the idiom identification tasks were carried out in both previous studies. On the one hand, in Liontas' (2002b) study, the target idioms for identification were embedded in a context of a few lines making up a paragraph at least, not in one continuous text and subjects knew that they just had to look for the idioms that each passage contained. In Dominguez (2008), idioms were presented in one running text just like in our study but subjects still were aware of the purposes of the task as they were explicitly told that they had to search the text and find the idioms present in it. Being aware of what they were looking for, subjects were able to find the hidden idioms as their attention was directed towards the purposes for which they had to go through the text. Although like in real life reading, Dominguez' (2008) study incorporated idioms within a text context as we did, nevertheless it is still a very much controlled task that doesn't have to do with naturalistic situations of reading material in English as a foreign language. In order to achieve this, subjects in our study were completely unaware that they were looking for idioms in the text as our initial aim was to see whether idioms could be identified by subjects as unknown units responsible for potential lexical problems that could lead to reading comprehension misunderstandings and misconceptions. Since the notion of noticing is considered to be a prerequisite of learning in SLA terms, we wanted to examine the extent to which L2 idioms are spontaneously spotted during their reading and so can be tackled as unknown lexical units via the use of lexical guessing strategies.

\subsubsection{Relationship between Idiom Identification Success and Idiom-type Variables}

Spearman rho correlations showed significant but negative correlations between success in the idiom identification task and just two of the idiom-type variables used in our investigation, namely, frequency of words in the idiom ( $\rho=-.495$, $p=.037)$ and interlingual similarity $(\rho=-.506, p=.032)$. These findings indicate 
that low frequency of the words within the idiom and low interlingual similarity helped learners identify the unknown phrasal idioms that were incorporated in the reading text.

\subsubsection{Relationship between Idiom Identification Success and Learner-related Variables}

Pearson correlations between success in the task of idiom identification in a text context and any of the individual difference variables included in this study yielded overall nonsignificant results.

However, level of the subjects in the private language school ('Frontistirio') they were attending was found to correlate significantly with success in idiom identification i.e. $Z=-2.122, p=.034$ suggesting that the higher level of L2 language proficiency subjects had attained in terms of the University of Cambridge EFL Examinations, the more readily they were to notice idioms in a text context. This finding might also possibly offer an explanation for the nearly $100 \%$ identification success rate of a few subjects in this study in that the more advanced the class they were attending opting for the certificates of CAE and CPE the more successful they were in identifying idioms during reading a text.

\subsection{Results for Idiom Comprehension Success}

\subsubsection{Overall Idiom Comprehension Success}

Similarly to the idiom identification success rate above but certainly to a far less extreme degree, overall success of idiom comprehension by Greek EFL learners during reading an English text is quite low with a mean of accurately inferred responses of $37.29 \%$ of the idioms subjects did not already know. Learners' attempts in the task of inferencing unknown phrasal idioms vary a lot $(S D=17.786)$ and are mainly clustered in the low and middle parts of the scale approximating reasonable well normal distribution curve as illustrated in figure 2. Thus, this finding demonstrates learners' difficulty in successfully inferring the meaning of the idioms they were not familiar with against the text context consciously since the target idioms were made known to the learners unlike the case in the L2 idiom identification task, and, consequently reveals their failure to overcome their lexical problems via the use of guessing strategies and further proceed with the task of reading comprehension in L2 English smoothly. 

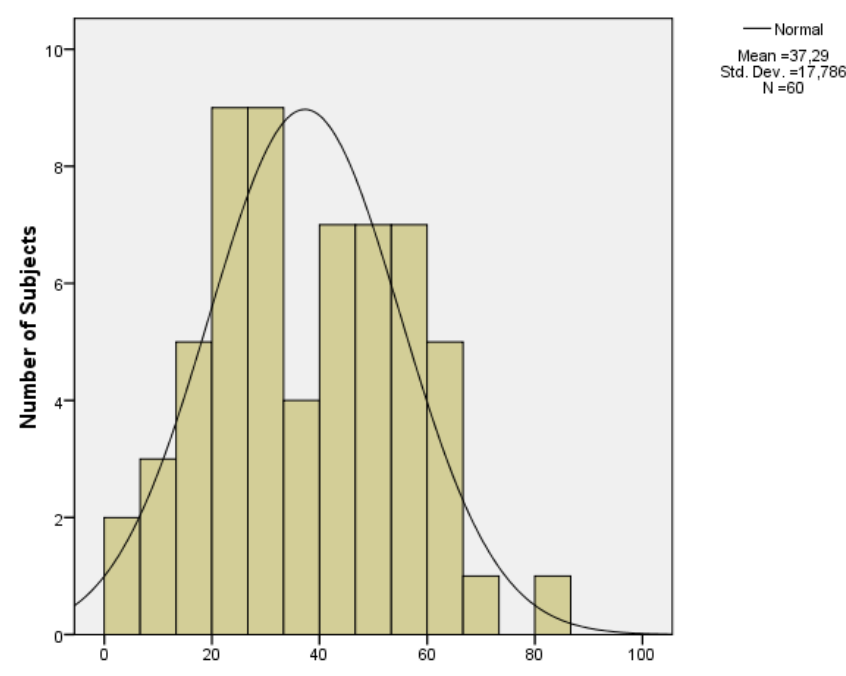

Percent of Success in Unknown Idiom Comprehension

Figure 2. Overall Success in the Idiom Comprehension Task of Unknown L2 Idioms through the use of Lexical Guessing Strategies (LGS)

This result illustrates a great difference between our case in Greece and L2 learners in different EFL contexts. Obviously our 37.29\% of L2 idiom comprehension success rate is not in line with the high percentages achieved in Liontas (2001) and Liontas (2002) where L2 learners' idiom comprehension success scores ranged from $86.42 \%$ when guessing at the meaning of Modern Greek idioms to $93.3 \%$ and $95 \%$ when guessing at the meaning of French and Spanish idioms respectively to as high as $100 \%$ in the case of learners guessing at the meaning of idioms in L2 German. Nevertheless, it should be pointed out that such discrepancy in overall L2 idiom comprehension results can also be due to the difference in the methodology followed in our study as compared to past research where the idioms that were to be guessed at were truly unknown to EFL learners and incorporated in a text context which at times might not have had enough helpful context for our L2 learners to guess from with accuracy as to the idioms' meaning.

\subsubsection{Relationship between Idiom Comprehension Success and Idiom-Type Variables}

On the other hand, success in the idiom comprehension task correlated significantly positively only with interlingual similarity $(\rho=.624, n=9, p<$ 0.01 , two tailed). This finding confirms Hypothesis 2 and is comparable to similar results produced by Irujo (1986) and Liontas (2001; 2002) where L1- 
L2 identical idioms in form and meaning were found to be the ones that were most frequently and successfully guessed at almost $100 \%$ in and out of context across Spanish, French, German and Modern Greek more than L1-L2 similar idioms and significantly more than L1-L2 totally different idioms.

Still, the overall idiom comprehension success for L2 English idioms with a Greek L1 idiom equivalence was found to be considerably lower, i.e. $48 \%$ as contrasted with $95 \%$ for L2 Spanish, $93.33 \%$ for L2 French, 100\% for L2 German and $86.42 \%$ for L2 Greek and the comprehension success rate for each of the idiom types included in our study was substantially different from that in the Liontas $(2001 ; 2002)$ studies: identical idioms $(67.6 \%)$ were also the ones guessed more easily than either similar (56.7\%) and different L1-L2 idioms (19.7\%) but to a far less extent than in Liontas $(2001 ; 2002)$ studies where success in comprehension reached almost $100 \%$ for identical and similar idioms in some languages.

Overall comprehension success of the nine English idioms of different levels of internal L2 semantic transparency appears considerably lower, i.e. 37.5\%. Transparent idioms were the most easily comprehended ones being guessed at a percent of $50.7 \%$ of the time, followed by the class of opaque idioms that were inferred accurately in $42.1 \%$ of the cases while semitransparent idioms seem to have been a cause of trouble for learners in their effort to understand their meaning within text context being comprehended successfully at a percent of only $19.8 \%$.

\subsubsection{Relationship between Idiom Comprehension Success and Learner-Related Variables}

Success in the task of idiom comprehension was also examined in relation to learners' L2 language proficiency, gender, motivation and field-independence/dependence. Overall, results revealed non-significant correlations between success in the task of idiom comprehension via the use of lexical guessing strategies in a text context and any of the main learner-related variables.

\section{Summary ofthe Main Findings and Implications for the EFL Classroom}

The results on L2 idiom identification success revealed a remarkably overall low ability on the Greek EFL learners' part to accurately identify unknown English phrasal idioms when reading a text for comprehension purposes. L2 idioms with no idiom equivalence in L1 Greek as well as idioms with low frequency word components were found to be identifiable most easily and successfully 
as compared to L1-L2 identical and similar idioms that were not noticed at all due to lack of salience. This finding highlights the importance of learner training in L2 idiom identification strategies raising their awareness with respect to the feature of 'deceptive transparency' of idioms as multiword lexical units have as well as to the potential problems that idioms' misidentification can present $\mathrm{L} 2$ learners with especially in the course of reading FL material. According to Nation (2001: 63-4), noticing is considered to be the first and the foremost step in the process of $L 2$ vocabulary acquisition followed by retrieval and creative use and can be most effectively achieved through decontextualization as an EFL teaching practice when the lexical item or the idiom under consideration is removed from its message context to be focused on as a language item.

Taking into consideration then that all language learning necessarily involves some degree of decontextualization, i.e. in order to acquire a foreign language, L2 learners need to consciously see language items as parts of the language system ('focus on form') rather than only as message then decontextualization in the case of $\mathrm{L} 2$ idioms can be implemented in the EFL classroom in the following three ways, i.e. (i) while listening or reading, the learner is guided, possibly through the use of special font or underlining, to notice an idiomatic phrase and is made aware of its salient feature of non-compositional meaning that does not correspond to the interpretation of the meaning of its individual component words. (ii) The learners can negotiate the form of an idiom with each other or with the teacher, i.e. learn to identify an idiom in terms of any special features with respect to its grammar, meaning and use. (iii) The teacher highlights an idiom and writes it on the blackboard giving emphasis to its semantic transparency or lack of it, equivalence in learners' L1, syntactic constraints and functional use in different texts and genres. (iv) The teacher explains a new L2 idiom to the learners by providing them explicitly with a definition, a first language translation or an L1 idiom equivalent. Explicit instruction on the key features of L2 idioms in terms of semantic transparency, syntactic flexibility and functional use will make FL learners' aware of idiom's formal characteristics and enable them to identify unknown target idioms more accurately in second language reading comprehension tasks.

With respect to success in L2 idiom comprehension, the results of this study revealed again an extremely low overall result that did not even reach 50\% of successful idiom meaning responses as provided by our Greek EFL learners, even when their attention was directed to try to guess them. The three idiom classes that were found to be most easily and successfully comprehended by L2 learners in this study were identical L1-L2 idioms, similar L1-L2 idioms and transparent English idioms indicating a possible learnability order for idioms in a foreign language. Thus, it could be suggested that with the exception of some common phrasal verbs that are already present in the lexical syllabus of beginner and lower intermediate levels, a selection of idioms with these characteristics 
could also be integrated within the syllabus of these levels familiarizing L2 learners with the notion of idiom in a FL and helping them build their $\mathrm{L} 2$ idiom knowledge incrementally starting from the early stages of their L2 education. Instruction of L2 idiomatic phrases can consist an integral part of any FL course by focusing on the appropriate types and the correct amount of $L 2$ idioms to be presented and acquired by learners at different stages of their foreign language learning concentrating first on idioms with high interlingual similarity and semantic transparency embedded in authentic reading text material. 


\section{References}

Dominguez, A.R. 2008. Identification, code-breaking and retention of L2 by EFL Mexican University students with more and less training. Unpublished PhD Thesis. University of Essex.

Fernando, C. and R. Flavell. 1981. On Idiom: Critical Reviews and Perspectives. Exeter Linguistic Studies, 5. Exeter: University of Exeter.

Irujo, S. 1986. Don't put your leg in your mouth: Transfer in the acquisition of idioms in a second language. TESOL Quarterly 20 (2), 287-304.

Laufer, B. 1989. A factor of difficulty in vocabulary learning: Deceptive transparency. AlLA Review 6, 10-20.

Laufer, B. 1997. The lexical plight in second language reading. In J. Coady and T. Huckin (Eds.), Second Language Vocabulary Acquisition: a Rationale for Pedagogy. Cambridge University Press, 20-34.

Laufer, B. and D.D. Sim. 1985. Taking the easy way out: Non-use and Misuse of clues in EFL reading. English Teaching Forum 23 (2), 7-10.

Liontas, J.I. 2001. That's All Greek to Me! The Comprehension and Interpretation of Modern Greek Phrasal Idioms. The Reading Matrix: An International Online Journal 1 (1), 1-32 (Available at: http//www.readingmatrix.com).

Liontas, J.I. 2002. Transactional Idiom Analysis: Theory and Practice. Journal of Language and Linguistics 1 (1), 17-53.

Makkai, A. 1972. Idiom Structure in English. The Hague: Mouton. 
Moon, R. 1998. Fixed Expressions and Idioms in English: A Corpus--based Approach. (Oxford Studies in Lexicography and Lexicology). Oxford: Oxford University Press.

Nattinger, J.R. 1988. Some current trends in vocabulary teaching. In R. Carter and M.J. McCarthy (Eds.) Vocabulary and Language Teaching. London: Longman, $62-82$

Nation, I.S.P. 2001. Learning Vocabulary in Another Language. Cambridge University Press.

Schmitt, N. 2000. Vocabulary in Language Teaching. Cambridge: Cambridge University Press.

Weinreich, U. 1969. Problems in the analysis of idioms. In J. Puhvel (Ed.), Substance and Structure of Language. Berkeley, California: University of California Press, 23-81. 


\section{Appendix}

\section{Idiom Identification Text}

Task 1

Read carefully the following text and:

1) While reading, underline words and/or phrases that you do not know.

2) Try to answer questions 1-7 below by choosing the answer ( $A, B, C$ or $D)$ which you think fits best according to the text.

Paul watched the television above the bar. Some turtles moved slowly across a beach in order to place a few smooth, white eggs in the small sand hills nearby. He saw the babies coming out dirty

5 from the broken shells and, this time, walking towards the rocks near the sea. Those who decided to cut the cord and not follow their family, they would surely be killed by sea birds. There was no pleasure in the cycle of birth and death. Yet the

10 turtles managed to survive because there were so many of them: one or two would disappear away into the sea unnoticed by the sea birds and save themselves. He asked himself why they went to all this trouble, though it could only be because they

15 had no choice. Their nature forced them to move on. If, by chance, a turtle could not give birth, then this turtle would die without reproducing itself. There was no escape for them. Once the wheels of reproduction were set in motion, it was

20 unstoppable.

At the present moment, his thoughts were driving him to sleep. If he had been one of those turtles, he would have lain down in the sand and simply waited for his death. Parenthood had taken

25 him by surprise and had definitely rocked the boat of his everyday routine where he was fighting to live from hand to mouth for most of the time. The books, the articles, the classes, had not prepared him for the intensity of it all. Now, everybody was

30 looking to him for the answers, blaming him for playing fast and loose with important decisions that could significantly affect his whole life. He flew off the handle when people criticized him for not making the correct decision. He had lived with

35 others all his life and knew that human relations was always a matter of throwing caution to the wind. For him, it was easy-you had fights but if they became too frequent or too boring, or if serious problems came up and you were no longer

40 happy, you just left, and tried again with someone else until you found someone you could put up with. But this time, things were different and he felt caught between a rock and a hard place, unable to get away from the difficult situation he was in.

45 He could not remember how it had all changed. Perhaps, it had been the demands that were suddenly being made of him to clean up his act and 
take up his responsibilities in the new reality, or even the faces turning towards him when a decision was required. Or perhaps it was just the intensity of his emotions together with the coffee and nicotine that added fuel to the fire and would not let him think clearly. Whatever it was, something had been forcing him to look for a quiet place in order to find some peace since last night.

Then, he smelt a rat: something nobody had warned him about. When he saw mother and baby together he realized that the reason why everyone talked about fatherhood these days 60 was because it was such an impossible state. Mothers and babies were riding high in the saddle. Fathers were optional extras. If men disappeared, the world would continue to exist and women would surely not turn a hair and would manage to find some way to reproduce. Within a generation or two it would be difficult to believe that there had ever been men at all. Little girls would try to understand what it had been that men had done, how they had

70 contributed. He had suddenly seen his role as that of a footnote and remembered the bad taste left in his mouth when he was told that he would become a father. The books had warned him of this feeling of jealousy. They had said it

75 was natural and that he would get over it, pick up the pieces, and move on once he stopped thinking about it. Fathers deceived themselves about their role in their children's upbringing. He could now see that that was like building

80 castles in the air, and that mothers and babies were everything. The men came and went causing trouble and trying to earn a crust to support their families in order to make up for it.

He turned his attention to the television. The

85 turtles he had watched moving towards the sea had become parents themselves now. They were the same turtles that had returned to the beach and placed their own eggs ready to take the rough with the smooth of becoming a parent. 


\section{What did Paul notice about the turtles in the first paragraph? [lines 1-8]}

A their unwillingness to return to the sea.

B their behavior with their young.

C the effort they made to survive.

D the small proportion of young who survived.

\section{Paul assumed that if a turtle did not wish to reproduce, [Par. 1. lines 8-13]}
A it would be punished by other turtles.
B it would end up doing so anyway.
C this attitude would not spread to other turtles.
D this would not come as a surprise.

\section{His thoughts turned towards going to sleep because [Par. 2, lines 13-17]}
A he knew that he was unlikely to get much in the near future.
$\mathbf{B}$ he had been tired of the thought that he would become a parent.
$\mathbf{C}$ he had become tired of his actions being criticized.
D he felt that that was what many of the turtles probably wanted to do.

\section{What did he feel he had been forced to do since last night? [Par. 3, lines}

\section{9-34]}
A accept that he was not really in his nature to live with other people.
B find a way of making himself feel better physically.
C identify precisely what had caused his life to change so radically.
D remind himself of how he had felt before this.

\section{In what way did he feel that he had been fooled? [Par. 4, lines 37-42]}

A He had expected his role to be one that differed from that of most men.

B He had not been informed about how women changed when they became mothers.

C He had not been told the truth by women about how they really regarded men.

D He had thought fatherhood was treated as a major topic because fathers were important. 
6. He felt that the books had failed to warn him that his feeling of jealousy [Par. 4, lines 46-50]

A would not disappear naturally.

B would not be shared by others.

C would be replaced by worse feelings.

D would make him be inactive.

\section{What is suggested about events on the television programme? [Par. 5]}

A They made him more depressed that he would otherwise have been.

B They made him feel that turtles were in a better state than humans were.

C They reflected his own lack of joy at becoming a father.

D They gave him a chance to escape from his own thoughts. 